\title{
Early death in acute promyelocytic leukemia: time to redefine risk groups
}

\author{
Meira Yisraeli Salman and Yishai Ofran \\ Department of Hematology, Shaare Zedek Medical Center, Jerusalem, Israel \\ E-mail: yofran@szmc.org.il \\ https://doi.org/10.3324/haematol.2021.280446
}

Acute promyelocytic leukemia (APL) stands out among its myeloid counterparts with unique clinical, morphological and cytogenetic elements. Sixty-five years ago, it was coined the "most malignant form of acute leukemia", with single digit remission rates and a median survival of 3.5 weeks. In 1973, a hallmark study by Bernard et al. ${ }^{2}$ described the exquisite sensitivity of APL to anthracyclines, rendering this fatal disease curable, although mortality remained high. The incorporation of all-trans retinoic acid (ATRA) and the later introduction of arsenic treatment significantly improved survival and gradually reduced the anxiety that a diagnosis of APL used to evoke among physicians. However, still to this day, early death rates, primarily due to severe coagulopathy, may reach $20-30 \%$.

Bernard and colleagues were also among the first to delineate prognostic factors important for survival, including elevated white blood cell (WBC) count and low levels of fibrinogen. Fast-forward to the year 2000, a risk-based model was being established, stratifying patients into three risk categories. ${ }^{3}$ The model, which became known as the Sanz risk model, defined high-risk patients as those with a WBC count above $10 \times 10^{\%} / \mathrm{L}$ at presentation, medium-risk patients as those with a WBC count less than $10 \times 10^{\%} / \mathrm{L}$ and a platelet count less than $40 \times 10^{9} / \mathrm{L}$ and low-risk patients as those with a WBC count less than $10 \times 10^{\circ} / \mathrm{L}$ and a platelet count of more than $40 \times 10^{\%} / \mathrm{L}$. However, considering the very low relapse rate in the current arsenic+ATRA era, survival risk scores should now be modified to concentrate on the risk of early death rather than relapse.

In this issue of Haematologica, Österroos et $a .^{4}$ set out to do just that, and present an elegant and simple scoring system for identifying APL patients most in danger of early death. Using real-world data from the Swedish AML registry, they created a model that is externally validated. The training cohort for the development of the prediction model included 301 consecutive adult patients diagnosed with APL in Sweden during the two decades leading up to 2020. The validation cohort encompassed 129 consecutive APL patients diagnosed at a university hospital in Portugal. The treatment protocols were slightly different between the two groups, but both were based on idarubicin induction plus oral ATRA until complete remission in the prearsenic era. From 2016 in the test cohort and 2018 in the validation one, arsenic replaced idarubicin for low- and intermediate-risk patients, and was added on for the high- risk ones. The score that was developed appears to predict early death more accurately than the Sanz criteria.

The three most important prognostic parameters that emerged from the analysis by Österroos and colleagues are WBC and platelet counts at diagnosis, as in the Sanz model, as well as age, which was not included in previous models. An additional and frankly, surprising, finding is that the point at which the risk of early death begins to rise may be from a WBC count as low as $2.2 \times 10^{\%} / \mathrm{L}$. The model stratifies patients into three WBC categories that correlate with risk; less than $3 \times 10^{9} / \mathrm{L}$, between $3-5 \times 10^{\%} / \mathrm{L}$ and more than $5 \times 10^{9} / \mathrm{L}$. Platelet counts are dichotomized with a cutoff of $30 \times 10^{\%} / \mathrm{L}$, and age is divided into four subgroups. The identification of the age contribution and the steep increase in risk with WBC counts between $2 \times 10 \% / L$ and $10 \times 10^{9} / \mathrm{L}$ are probably the main contributing factors to the score's sensitivity.

The score has clear, potentially practice-changing implications. Preventing early death by applying immediate aggressive supportive measures to all patients at high risk is imperative. Thus, identification of high-risk patients "hiding" among patients who were previously incorrectly considered to be of low risk, can improve patient care and save lives. Other suggested risk models focus primarily on bleeding risk, ${ }^{5}$ while this model encompasses all early death risks.

To best apply this new risk model in clinical practice, one should focus on the risk calculator presented in Figure 3 in the paper by Österroos et al. ${ }^{4}$ We recommend that all patients who present with a risk of early death $>10 \%$ be treated with aggressive supportive measures. According to the proposed model, these high and very-high risk groups include all patients with a WBC count greater than $5 \times 10^{9} / L$ as well as those with lower WBC counts but with advanced age.

The presented scoring system has several compelling features. Based on real-world data, the cohorts had no exclusion criteria and included all patients, even those who were too ill or too old to be included in prospective clinical trials, thus greatly improving the reliability of the data. The authors also developed an easy-to-use online tool, readily available for any clinician to use at the bedside in the Emergency Department.

The fact that age emerged as an important factor predicting early death is worth dwelling on. It is well known that age is a major factor both in acute leukemia pathogenesis 
and prognosis, and in pathologies of the coagulation system. It would be interesting to study whether it is the effect of aging on leukemia or on the coagulation system itself which contributes to the increase in early death risk.

As the authors stated, only a small percentage of patients in the cohort received the combination of arsenic with ATRA, as this treatment became prevalent only late during the study period. In addition, future modifications of this score beg inclusion of molecular data if these could be acquired early after diagnosis. One more point to consider may be the inclusion of general coagulopathy risk factors, such as those known to be involved in veno-occlusive diseases, in the scoring system. ${ }^{6}$ Clearly more validation is necessary in order to create clinical guidelines based on this score, primarily relating to increased surveillance and to recommending risk-adapted thresholds for platelet, cryoprecipitate and/or fresh-frozen plasma transfusions. To conclude, Österoos and colleagues developed a practical and effective score that may allow identification of highrisk patients hidden among those previously classified as low risk. Aggressive supportive care, if applied, can save the lives of these patients. Perhaps the entire risk-stratification system in APL that is currently guiding treatment should be revamped, and focus on risk of early death. Disregarding age as an important prognostic factor should be remedied, and the WBC cutoff, if used, should be age dependent.

\section{Disclosures}

No conflicts of interest to disclose.

\section{Contributions}

MYS and YO co-wrote the manuscript

\section{References}

1. Hillestad LK. Acute promyelocytc leukemia . Acta Med Scand.1957;159(3):189-194.

2. Bernard J, Weil M, Boiron M, et al. Acute promyelocytic leukemia: results of treatment by daunorubicin. Blood. 1973;41(4):489-496.

3. Sanz MA, Lo Coco F, Martin G, et al. Definition of relapse risk and role of nonanthracycline drags for consolidation in patients with acute promyelocytic leukemia: a joint study of the PETHEMA and GIMEMA cooperative groups. Blood. 2000;96(4):1247-1253.
4. Österroos A, Maia T, Eriksson A, et al. A risk score based on realworld data to predict early death in acute promyelocytic leukemia. Haematologica. 2022;107(7):1528-1537.

5. Mitrovic M, Suvajdzic N, Bogdanovic A, et al. International Society of Thrombosis and Hemostasis Scoring System for disseminated intravascular coagulation $\geq 6$ : a new predictor of hemorrhagic early death in acute promyelocytic leukemia. Med Oncol. 2013;30(1):478.

6. Mitrovic M, Suvajdzic N, Elezovic I, et al. Thrombotic events in acute promyelocytic leukemia. Thromb Res. 2015;135(4):588-593. 